\section{Transcriptional integration of mitogenic and mechanical signals by Myc and YAP}

\author{
Ottavio Croci, ${ }^{1,5}$ Serena De Fazio, ${ }^{1,5}$ \\ Francesca Biagioni, 1,5 Elisa Donato, 1,4,5 \\ Marieta Caganova, ${ }^{1}$ Laura Curti, ${ }^{1}$ Mirko Doni, ${ }^{2}$ \\ Silvia Sberna, ${ }^{1}$ Deborah Aldeghi, ${ }^{1}$ \\ Chiara Biancotto, ${ }^{1}$ Alessandro Verrecchia, ${ }^{2}$ \\ Daniela Olivero, ${ }^{3}$ Bruno Amati, ${ }^{1,2}$ \\ and Stefano Campaner ${ }^{1}$
}

\begin{abstract}
${ }^{1}$ Center for Genomic Science of IIT@SEMM (Istituto Italiano di Tecnologia at European School of Molecular Medicine), Fondazione Istituto Italiano di Tecnologia (IIT), 20139 Milan, Italy; ${ }^{2}$ Department of Experimental Oncology, European Institute of Oncology (IEO), 20139 Milan, Italy; ${ }^{3}$ Laboratorio di Analisi Veterinarie BiEsseA, 20129 Milan, Italy
\end{abstract}

Mammalian cells must integrate environmental cues to determine coherent physiological responses. The transcription factors Myc and YAP-TEAD act downstream from mitogenic signals, with the latter responding also to mechanical cues. Here, we show that these factors coordinately regulate genes required for cell proliferation. Activation of Myc led to extensive association with its genomic targets, most of which were prebound by TEAD. At these loci, recruitment of YAP was Myc-dependent and led to full transcriptional activation. This cooperation was critical for cell cycle entry, organ growth, and tumorigenesis. Thus, Myc and YAP-TEAD integrate mitogenic and mechanical cues at the transcriptional level to provide multifactorial control of cell proliferation.

Supplemental material is available for this article.

Received April 28, 2017; revised version accepted October 13, 2017.

Cell cycle entry in higher eukaryotes depends on extracellular cues mediated by growth factors, metabolites, cell adhesion, and cell-cell contacts. These signals must be interpreted and integrated by cells to regulate complex gene expression programs. Mitogenic agents, such as serum growth factors, stimulate cell proliferation by activating waves of transcription, starting with immediate early genes, which in turn regulate the expression of delayed early genes. c-myc is an immediate early gene whose product, the transcription factor (TF) Myc, is essential for se-

[Keywords: YAP; TEAD; Myc; transcription; Hippo signaling] ${ }^{4}$ Present address: Division of Stem Cells and Cancer, Deutsches Krebsforschungszentrum (DKFZ), Heidelberg Institute for Stem Cell Technology and Experimental Medicine (HI-STEM gGmbH), 69120 Heidelberg, Germany.

${ }^{5}$ These authors contributed equally to this work. Corresponding author: stefano.campaner@iit.it

Article published online ahead of print. Article and publication date are online at http://www.genesdev.org/cgi/doi/10.1101/gad.301184.117. Freely available online through the Genes \& Development Open Access option. rum-mediated and growth factor-mediated cell cycle entry (Kelly et al. 1983; Armelin et al. 1984; Roussel et al. 1991; Barone and Courtneidge 1995; de Alboran et al. 2001; Trumpp et al. 2001; Perna et al. 2012). This function of Myc stems from its ability to control the expression of a large fraction of genes involved in cell activation and proliferation.

When ectopically expressed in quiescent cells, Myc is able to drive cell cycle progression in the absence of serum (Eilers et al. 1991; Pelengaris et al. 1999). This effect of Myc is context-dependent, however, since not all cells or tissues respond to Myc by entering the cell cycle (Jackson et al. 1990; Xiao et al. 2001; Murphy et al. 2008). This suggests that a full proliferative response may require the engagement of other TFs, which may respond to different regulatory signals, such as metabolic or mechanical cues. Recently, YAP has emerged as a key TF in the control of cell growth and organ size in response to a variety of signals such as cell adhesion, apico-basolateral polarity, cytoskeletal tension, and mitogens. YAP activity is controlled by a cascade of regulatory kinases, the Hippo pathway, and mechanotransduction: When either Hippo signaling is low or in conditions of high cytoskeletal tension, YAP translocates into the nucleus, where it associates with TEAD TFs to regulate transcription (Vassilev et al. 2001).

Here we show that YAP coadjuvates Myc-dependent transcription and cooperates in inducing cell cycle entry and cell proliferation both in vitro and in vivo. This depends on the constitutive binding of TEAD to a large fraction of Myc target genes. At these promoters, Myc binding was independent of YAP and led to increases in the histone methylation mark $\mathrm{H} 3 \mathrm{~K} 4 \mathrm{me} 3$. While this was insufficient for full transcriptional activation, it converted these loci into high-affinity binding sites for YAP. Thus, in low Myc conditions, YAP was bound to its canonical targets, was redistributed to a fraction of Myc target genes upon Myc accumulation, and favored activation of those loci. This multilayered circuit explains how Myc can selectively control gene expression despite its extensive genomic interactions (Kress et al. 2015) and how the modulation of Myc transcriptional programs in cis by other TFs allows proper integration of diverse mitogenic stimuli.

\section{Results and Discussion}

To dissect Myc-induced proliferation, we used 3T9 ${ }^{\text {MycER }}$ fibroblasts, which constitutively express MycER ${ }^{\mathrm{TM}}$, a chimeric protein that can be conditionally activated by 4 hydroxytamoxifen (OHT) (Littlewood et al. 1995). As reported (Eilers et al. 1991; Barone and Courtneidge 1995), activation of MycER in serum-starved subconfluent cells was sufficient to stimulate cell cycle entry and Myc-dependent transcription (Fig. 1A; Supplemental Fig. S1A, B). However, these effects were strongly inhibited by cellular confluence (Fig. 1A; Supplemental Fig. S1A,B), suggesting that cell-cell contact and low cytoskeletal tension are inhibitory to Myc-driven proliferation.

(C) 2017 Croci et al. This article, published in Genes \& Development, is available under a Creative Commons License (Attribution 4.0 International), as described at http://creativecommons.org/licenses/by/4.0/. 
A

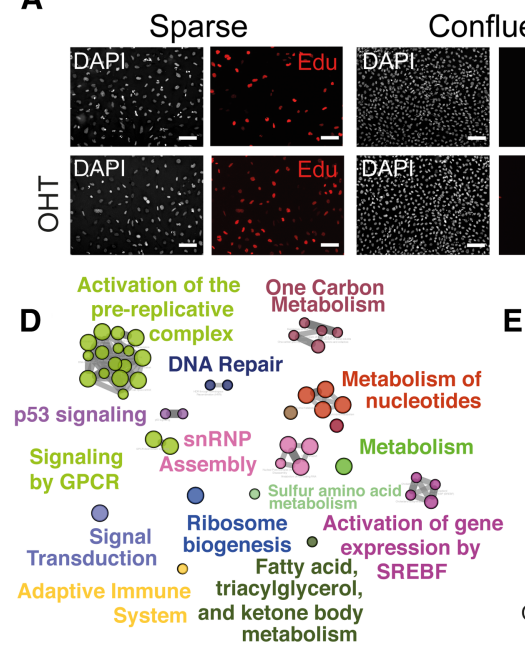

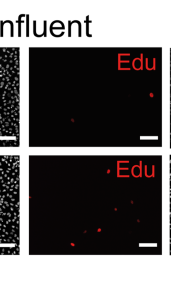

E

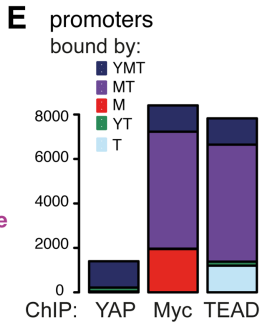

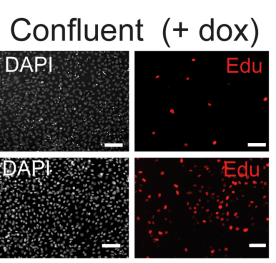

$-100 \mu \mathrm{m}$

F Myc peaks

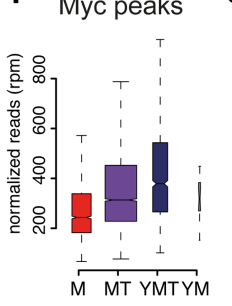

B

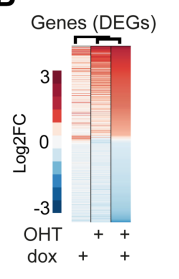

G YAP peaks

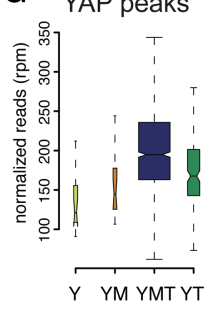

C

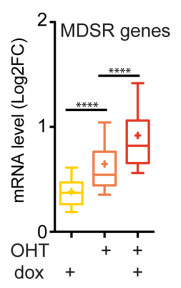

H YMT bound genes

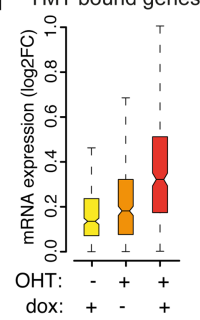

Figure 1. Myc and YAP coregulate cell cycle entry. Serum-starved subconfluent (sparse) $(A)$ or highly confluent (confluent) $3 \mathrm{~T} 9^{\mathrm{MycER} ; \mathrm{YAP}}(B-H)$ cells were treated with OHT to activate MycER and doxycycline (dox) to trigger the expression of YAP ${ }^{\mathrm{S} 127 \mathrm{~A}}$. $(A)$ Cell cycle entry was measured by immunofluorescence analysis of EdU incorporation. DAPI was used to color nuclei. $(B)$ Ranked heat map based on the $\log _{2}$ fold change of the differentially expressed genes (DEGs) identified by RNA sequencing (RNA-seq). (C) Box plot of the mRNA expression level of the Myc-dependent serum response (MDSR) genes $(D)$ Gene ontology map based on the DEGs determined upon both MycER activation and YAP induction. (E) Cumulative bar graph of Myc, YAP, and TEAD ChIP-seq (chromatin immunoprecipitation [ChIP] combined with high-throughput sequencing) peaks, colorcoded based on their overlap. (Y) YAP; $(\mathrm{M})$ Myc; $(\mathrm{T})$ TEAD. $(F, G)$ Box plot of the enrichment of Myc $(F)$ and YAP $(G)$ ChIP-seq peaks divided into subsets as in $E$. $(H)$ Expression levels of up-regulated genes cobound at their promoters by YAP, Myc, and TEAD (YMT peaks).

While Myc controls cell cycle entry in response to mitogenic signals, YAP also regulates cell proliferation in response to mechanical and cytoskeletal cues (Zhao et al. 2007; Dupont et al. 2011; Fernandez et al. 2011; Sansores-Garcia et al. 2011; Wada et al. 2011; Halder et al. 2012; Aragona et al. 2013). To address whether YAP could cooperate with MycER in cell cycle entry, we transduced $3 T 9^{\text {MycER }}$ cells with a doxycycline-inducible vector expressing the activated mutant $\mathrm{YAP}^{\mathrm{S} 127 \mathrm{~A}}$ (referred to here as $3 \mathrm{~T}^{\mathrm{MycER} ; \mathrm{YAP}}$ cells) (Supplemental Fig. S1C; Zhao et al. 2007). While either MycER or YAP ${ }^{\mathrm{SY2} 7 \mathrm{~A}}$ alone had no significant effect in serum-starved confluent cells, their coactivation resulted in robust cell cycle entry (Fig. 1A; Supplemental Fig. S1D,E). This was paralleled by the differential expression of a large number of genes (DEGs [differentially expressed genes]) that responded to MycER and YAP together but not-or less significantly - to either alone (Fig. 1B; Supplemental Fig. S2A-D). These genes were linked mainly to cell proliferation (Fig. 1E; Supplemental Fig. S2E,F) and included previously identified Myc-dependent serum response (MDSR) genes (Fig. 1C; Supplemental Fig. S2D; Perna et al. 2012). In a general manner, the response of MycER-induced genes was augmented by coactivation of YAP (Fig. 1C; Supplemental Fig. S2D).

This was not due to reciprocal regulation of MycER and YAP protein levels or nuclear localization, which were largely unaffected by their coexpression both in vitro and in vivo (Supplemental Figs. S1C, S3A-G). Instead, MycER activation increased the chromatin-associated fraction of YAP (Supplemental Fig. S3H).

Consistent with the above, ChIP-seq (chromatin immunoprecipitation [ChIP] combined with high-throughput sequencing) analysis revealed extensive overlaps in the genomic localization of YAP, TEAD, and Myc (YMT) (Supplemental Fig. S4,A-C). In particular, virtually all YAPassociated promoters were co-occupied by TEAD and
Myc (Fig. 1E). Genomic sites cobound by the three proteins (i.e., YMT peaks) showed the greatest enrichment for each single TF (Fig. 1F, G; Supplemental Fig. S4D,E). Genes cobound at their promoters by the three TFs showed stronger transcriptional responses to YAP and Myc together than to either TF alone (Fig. 1H). Thus, YAP and Myc coregulate a subset of Myc target genes, in particular those linked to cell cycle entry.

In sparse cultures, where high cytoskeletal tension activates YAP (Zhao et al. 2007; Dupont et al. 2011; Fernandez et al. 2011; Sansores-Garcia et al. 2011; Wada et al. 2011; Halder et al. 2012; Aragona et al. 2013), YAP inhibitors blocked Myc-induced cell cycle entry and transcription of Myc target genes (Fig. 2A,B; Supplemental Figs. S5, S6). Similarly, YAP deletion impaired the expression of Myc target genes (Fig. 2C). In the same conditions, pharmacological relief of cytoskeletal tension (with blebbistatin, an inhibitor of actomyosin contraction) or blockade of actin-mediated signaling (with the ROCK inhibitor Y276632) impaired both Myc-induced cell cycle entry and induction of Myc target genes, which were rescued by overexpression of an activated YAP mutant $\left(\mathrm{YAP}^{\mathrm{S} 127 / 38 \mathrm{AA}}\right.$ ) (Fig. 2D,E; Supplemental Fig. S7). Overall, these data suggest that Myc-induced transcription and cell cycle entry rely on the activation of endogenous YAP by cytoskeletal tension. In line with this, coexpression of $\mathrm{YAP}^{\mathrm{S} 127 \mathrm{~A}}$ promoted anchorage-independent growth of MycER cells (a growth condition where cytoskeletal tension is low) while not providing any proliferative advantage in two-dimensional conditions (Fig. 2F; Supplemental Fig. S8).

We then addressed the relevance of our finding in a postmitotic adult tissue. Toward this aim, we used mouse strains allowing doxycycline-inducible expression of Myc (tet-Myc) (Beer et al. 2004; Shachaf et al. 2004; Kress et al. 2016) and YAP (tet-YAP) (Jansson and Larsson 2012) in the liver. While short-term induction (48 h) of either 

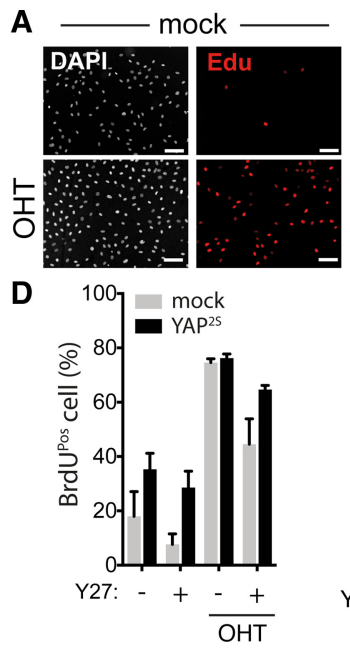
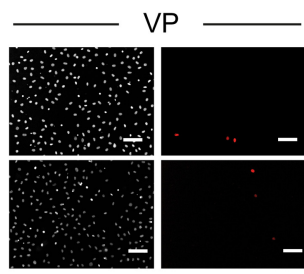

B

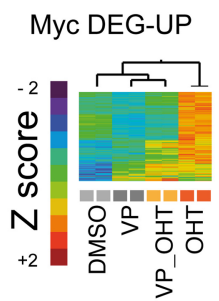

E

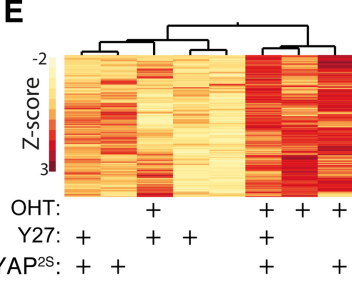

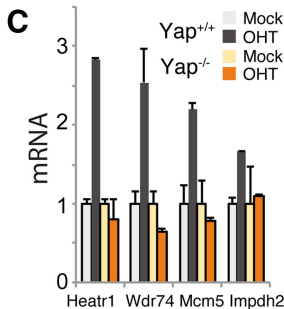

$\mathbf{F}$
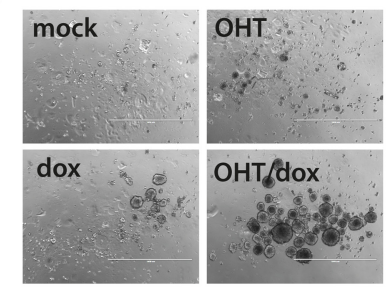

Figure 2. Myc-driven cell cycle entry depends on YAP activity and cytoskeletal tension. $(A-E)$ Serum-starved subconfluent fibroblasts were kept in low serum and treated as indicated. (A) Immunofluorescence analysis of Myc-induced cell cycle entry of $3 \mathrm{~T}^{\mathrm{MycER}}$ measured as EdU incorporation on cells treated with the YAP inhibitor verteporfin (VP). $(B)$ Expression analysis (clustering) of Myc up-regulated genes following VP treatment. (C) RT-qPCR expression of Myc target genes in MycER fibroblasts either wild type $\left(Y^{\prime} P^{+/}\right)$or knockout (Yap ${ }^{-/-}$) for Yap. (D) S-phase entry by BrdU incorporation (by FACS) in MycER fibroblasts overexpressing YAP ${ }^{\mathrm{S127A} / \mathrm{s} 318 \mathrm{~A}}$. Cells were treated with OHT to activate MycER and with the ROCK inhibitor Y276632 (Y27) as indicated. $(E)$ Clustered heat map of normalized mRNA expression of cells shown in $D$. $(F)$ Anchorage-independent growth assay of bipotential mouse embryonic liver (BMEL) cells overexpressing MycER and tet-YAP ${ }^{\mathrm{S} 127 \mathrm{~A}}$, treated as indicated. Representative pictures of cell colonies are shown.

Myc or YAP alone led to a mild proliferative response, their coactivation (tet-Myc/YAP) resulted in robust cellular proliferation (Fig. 3A; Supplemental Fig. S9). Genomewide expression analysis by RNA sequencing (RNA-seq) revealed a large number of genes (2500) coregulated by YAP and Myc but not by either factor alone (Fig. 3B,C;
Supplemental Fig. S10A-E). These genes were enriched for ontological terms linked mainly to cell proliferation and included the aforementioned MDSR genes (Supplemental Fig. S11). On the other hand, YAP target genes were down-modulated by coexpression of Myc (cluster 6) (Supplemental Fig. S10E,F), consistent with the reported

\section{A}
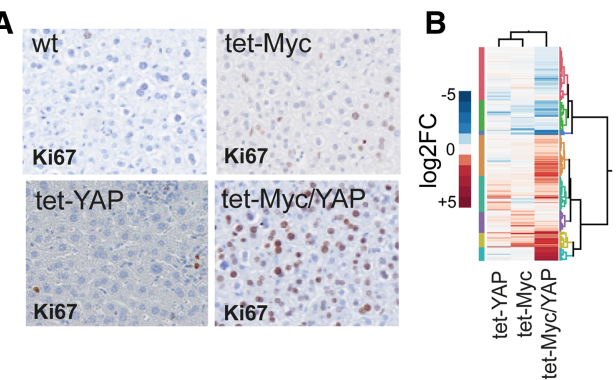

E

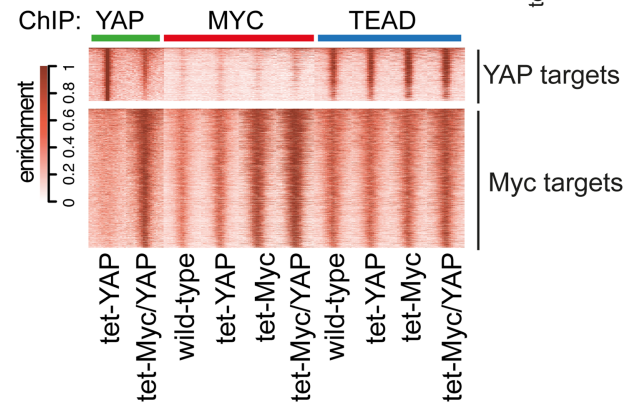

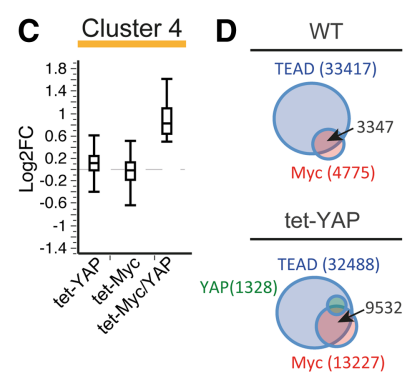

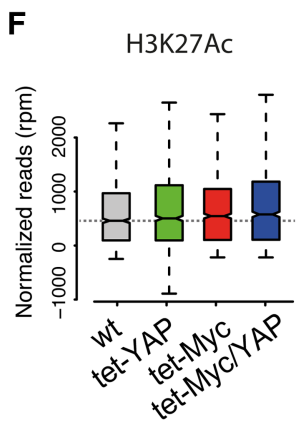

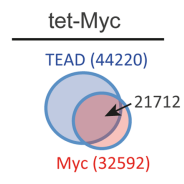

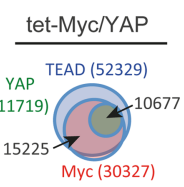

G

H3K4me3

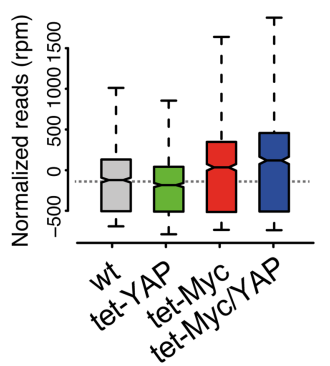

Figure 3. Cooperative binding and transcriptional activation by Myc, TEAD, and YAP. Genome-wide analyses of livers from R26-rtTA mice either wild type (wt), transgenics for Myc (tet-Myc) or YAP (tet-YAP), or double transgenics (tet-Myc/YAP). Short-term induction was achieved by feeding mice with doxycycline-containing food for $48 \mathrm{~h}$. $(A)$ Liver sections stained with an anti-Ki67 antibody. $(B)$ Hierarchical clustering of DEGs. $(C)$ Box plot showing a representative cluster of YAP/Myc DEGs. (D) Venn analysis of Myc, YAP, and TEAD ChIP-seq peaks. The number of peaks determined for each TF is reported in brackets; the arrows point to the number of overlapping peaks. $(E)$ Ranked heat maps of the ChIP-seq enrichment of the indicated TFs. (Top panel) YAP peaks detected only in tet-YAP livers. (Bottom panel) Promoters bound by YAP in tet-Myc/YAP livers. $(F, G) \mathrm{H} 3 \mathrm{~K} 27 \mathrm{ac}(F)$ and $\mathrm{H} 3 \mathrm{~K} 4 \mathrm{me} 3(G)$ levels at promoters of DEG-up genes cobound by Myc and YAP. 
Croci et al.
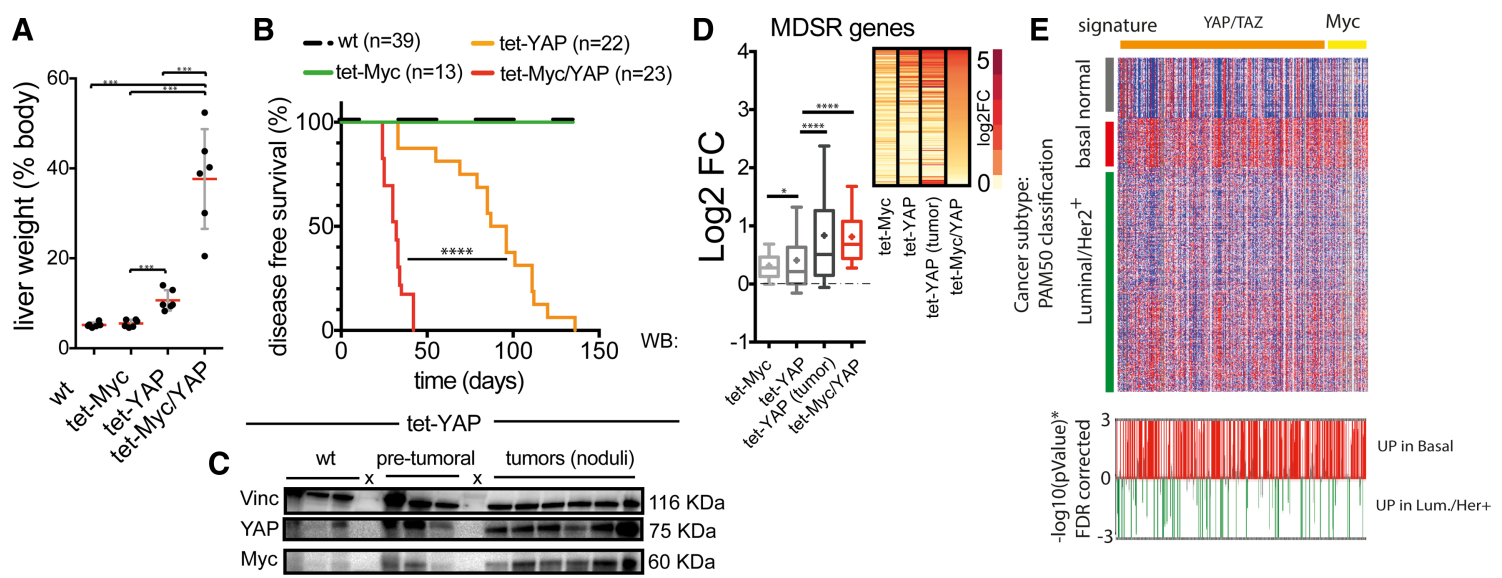

Figure 4. Myc and YAP cooperate in inducing liver growth and tumorigenesis. $(A)$ Liver weight assessed at 5 wk of induction. Data are reported as percentage relative to total body weight. $(B)$ Kaplan-Meier disease-free survival analysis. (C) Western blotting analysis of YAP and Myc levels in LAP-tTA tet-YAP mice at the pretumoral stage (4 wk of YAP activation) and in tumors. Vinculin (vin) was used as aninternal control for equal loading. $(D)$ Box plot of the expression level of MDSR genes up-regulated in the liver upon YAP and/or Myc induction. (Inset at the right) Ranked heat map. (E, top panel) Heat map of Myc and YAP/TAZ gene signatures based on the expression data of breast cancers (TCGA_BRCA). The heat map was clustered by breast cancer subtypes (basal-like, normal-like, and Luminal/Her2 ${ }^{+}$). (Bottom panel) The statistical track shows the logarithmic plot of $P$-values for each gene. (Red bars) Genes up in basal-like; (green bars) genes up in Luminal/Her ${ }^{+}$.

antagonism between Myc and YAP signaling (von Eyss et al. 2015).

We then profiled the genomic interactions of Myc, YAP, and TEAD by ChIP-seq in the liver. While Myc displayed limited chromatin association in wild-type hepatocytes (only 5000 peaks), its short-term induction led to extensive chromatin binding (>30,000 peaks). A comparable increase of Myc peaks was detected in tet-Myc/YAP livers, indicating that Myc binding to chromatin depended on its expression level but not on YAP expression (Fig. 3D; Supplemental Fig. S12A). TEAD showed widespread chromatin interactions with a similar genomic distribution among the four experimental groups (Fig. 3D; Supplemental Fig. S12A). YAP showed no significant chromatin association in either wild-type or tet-Myc mice, consistent with its low expression level (Supplemental Figs. S3F, S12A). However, YAP peaks became detectable in tet-YAP and were further boosted in tetMyc/YAP mice in both number and level of enrichment (Fig. 3D,E; Supplemental Fig. S12A,B). Importantly, in tet-YAP livers, YAP showed only a partial genomic overlap with Myc, while coexpression in tet-Myc/YAP favored its recruitment to genomic sites bound by both Myc and TEAD (Fig. 3D,E). Thus, Myc caused a global shift in the genomic distribution of YAP, favoring its recruitment to promoters bound by both Myc and TEAD (Fig. 3E, bottom panel; Supplemental Fig. S12C) and its concomitant decrease from the canonical TEAD/YAP targets most significantly enriched in the livers of tet-YAP mice (Fig. 3E, top panel). This reshuffling of YAP away from its canonical binding sites provides a rationale for the repression of YAP target genes that we and others (von Eyss et al. 2015) observed upon Myc activation.

In tet-Myc/YAP mice, genomic loci cobound by YMT peaks showed stronger enrichment of all TFs compared with regions bound by each TF alone (Supplemental Fig. S12D). Likewise, genomic loci cobound by YMT in tetMyc/YAP livers had the strongest enrichment for each TF when both Myc and YAP were overexpressed compared with all other conditions (Fig. 3E; Supplemental
Fig. S12E). Importantly, a consistent number of genes differentially expressed in tet-Myc/YAP cells were cobound at their promoters by YMT (Supplemental Fig. S12F).

Myc generally requires a pre-existing active chromatin environment to access DNA, characterized in particular by the histone marks H3K4me3 and H3K27ac (Guccione et al. 2006; Sabo et al. 2014; Kress et al. 2015); indeed, in wild-type livers, these marks pre-existed on the promoters of Myc/YAP coregulated genes (Supplemental Fig. S12G, $\mathrm{H})$. At these loci, H3K27ac was slightly increased by binding of YAP, Myc, or both (Supplemental Fig. S12G). $\mathrm{H} 3 \mathrm{~K} 4 \mathrm{me} 3$ was low in wild type and tet-YAP but increased upon Myc binding and was further enhanced by YAP, implying a role of TF-induced chromatin modifications in stabilizing the YMT complex on these sites (Fig. 3F; Supplemental Fig. S12H; Stein et al. 2015). This suggested cooperative binding of these TFs, with Myc and TEAD favoring YAP recruitment to their common target loci.

Next, we wondered whether induction of Myc and YAP might also have long-term consequences on liver growth. As reported (Camargo et al. 2007; Dong et al. 2007), tetYAP mice developed mild hepatomegaly within $5 \mathrm{wk}$ of induction (Fig. 4A), indicating that sustained activation of YAP in the liver can result in a proliferative response that is likely due to the engagement of secondary cellular programs (Camargo et al. 2007; Yimlamai et al. 2014). tetMyc mice showed no signs of liver enlargement. Instead, coinduction of Myc and YAP led to massive hepatomegaly, which accounted for the remarkably short disease-free survival of these mice (Fig. 4A,B; Supplemental Fig. S13A). Histologically, these livers were classified as hepatocellular carcinomas with a diffuse solid pattern of growth, indicating pervasive aberrant proliferation (Supplemental Fig. S13B-D).

As reported (Camargo et al. 2007; Dong et al. 2007), prolonged activation of YAP alone led to the development of focal tumor lesions with full penetrance. This was paralleled by a progressive elevation of Myc levels, which peaked in tumoral lesions (Fig. 4C,D; Supplemental Fig. S14AC). MDSR genes were progressively up-regulated to reach 
levels in tumors that were comparable with those observed in tet-Myc/YAP mice (Fig. 4D). RNA-seq analysis showed similarities among YAP-driven tumors and tetMyc/YAP livers, both of which clustered apart from tetMyc or tet-YAP alone (Supplemental Fig. S14D). These data suggest a selective pressure for the up-regulation of Myc in YAP-driven tumors, reiterating the strong mitogenic effect observed upon coactivation of both oncogenes. Accordingly, the Myc and Hippo gene signatures were coenriched in basal-like breast tumors (Fig. 4E), a subset with reported deregulation of the Hippo pathway (Cordenonsi et al. 2011), and both the YAP/TAZ and Myc signatures could independently stratify basal-like tumors (Supplemental Fig. S15).

In summary, we described here a cis-regulatory network comprising Myc and the YAP-TEAD complex that controls the expression of proliferative genes. The promoters of genes activated by Myc and YAP were prebound by TEAD, were heavily bookmarked by chromatin activation marks, and showed stalled RNA polymerase II (Supplemental Fig. S12I), indicating that they were poised for activation. Myc binding was YAP-independent, although the presence of YAP favored further stabilization of Myc on chromatin. On the other hand, recruitment of YAP to Myc-TEAD-bound promoters was fully dependent on prebound Myc. Our data suggest that YAP recruitment on Myc sites is favored by Myc-induced epigenetic remodeling (which increases H3K4me3 and, to a lower extent, $\mathrm{H} 3 \mathrm{~K} 27 \mathrm{ac}$ levels) as well as protein-protein interaction between Myc and YAP (Supplemental Fig. S16). Further work will be needed to fully dissect the molecular mechanism of YAP recruitment to these loci.

Altogether, our findings provide a parsimonious solution for the integration of mitogenic and mechanical signals in the control of cell cycle entry and proliferation. Our data also illustrate how convergent signals (here, YAP activation) endow Myc with the ability to control selective transcriptional responses in spite of its pervasive association with the genome (Perna et al. 2012; Sabo et al. 2014; Walz et al. 2014; Kress et al. 2015; Piccolo et al. 2017).

\section{Materials and methods}

\section{Mouse strains}

Tet-YAP mice (Col1A1-YAP ${ }^{S 127 A}$ transgenic mice) were kindly provided by Dr. Jonas Larsson. tet-MYC transgenic mice were a kind gift from Dr. Martin Eilers. For liver-specific transgene expression, mice were crossed with LAP-tTA mice expressing the tTA tetracycline transactivator under the control of the LAP promoter [B6.Cg-Tg(tTALap $\mid 5 \mathrm{Bj} \mathrm{d} / \mathrm{J}$; purchased from Jackson Laboratories].

\section{Cell culture}

3T9 ${ }^{\text {MycER }}$ murine fibroblasts (Sabo et al. 2014) were infected with pSlik$\mathrm{YAP}^{\mathrm{S} 127 \mathrm{~A}}$ retroviruses and selected with $100 \mathrm{\mu g} / \mathrm{mL}$ hygromycin. MycER was activated by the addition of $20-400 \mathrm{nM}$ OHT, while $\mathrm{YAP}^{\mathrm{S} 127 \mathrm{~A}}$ was induced by $2 \mu \mathrm{g} / \mathrm{mL}$ doxycycline. Detailed experimental procedures and data analysis are in the Supplemental Material.

RNA-seq and ChIP-seq data have been deposited in NCBI's Gene Expression Omnibus (GEO) and are accessible through GEO series accession number GSE83869.

\section{Acknowledgments}

We thank Dr. M. Eilers, Dr. J. Larsson, and Dr. L. Jansson, for kindly sharing reagents; Dr. G. Natoli, Dr. A. Sabò, Dr. A. Bisso, Dr. S. Piccolo, Dr. F.
Zanconato, and Dr. T. Kress for discussion and suggestions; Salvatore Bianchi, Thelma Capra, and Luca Rotta for technical support in next-generation sequencing; and Paola Nicoli for technical assistance. This work was supported by the Italian Association for Cancer Research (AIRC, IG grant 13135 ) to S.C., and grants from the Italian Health Ministry (RF2011-02346976) and the Italian Association for Cancer Research (AIRC 2015-16768) to B.A.

\section{References}

Aragona M, Panciera T, Manfrin A, Giulitti S, Michielin F, Elvassore N, Dupont S, Piccolo S. 2013. A mechanical checkpoint controls multicellular growth through YAP/TAZ regulation by actin-processing factors. Cell 154: 1047-1059.

Armelin HA, Armelin MC, Kelly K, Stewart T, Leder P, Cochran BH, Stiles CD. 1984. Functional role for c-myc in mitogenic response to plateletderived growth factor. Nature 310: 655-660.

Barone MV, Courtneidge SA. 1995. Myc but not Fos rescue of PDGF signalling block caused by kinase-inactive Src. Nature 378: 509-512.

Beer S, Zetterberg A, Ihrie RA, McTaggart RA, Yang Q, Bradon N, Arvanitis C, Attardi LD, Feng S, Ruebner B, et al. 2004. Developmental context determines latency of MYC-induced tumorigenesis. PLOS Biol 2: e332.

Camargo FD, Gokhale S, Johnnidis JB, Fu D, Bell GW, Jaenisch R, Brummelkamp TR. 2007. YAP1 increases organ size and expands undifferentiated progenitor cells. Curr Biol 17: 2054-2060.

Cordenonsi M, Zanconato F, Azzolin L, Forcato M, Rosato A, Frasson C, Inui M, Montagner M, Parenti AR, Poletti A, et al. 2011. The Hippo transducer TAZ confers cancer stem cell-related traits on breast cancer cells. Cell 147: 759-772.

de Alboran IM, O'Hagan RC, Gartner F, Malynn B, Davidson L, Rickert R, Rajewsky K, DePinho RA, Alt FW. 2001. Analysis of C-MYC function in normal cells via conditional gene-targeted mutation. Immunity 14: 45-55.

Dong J, Feldmann G, Huang J, Wu S, Zhang N, Comerford SA, Gayyed MF, Anders RA, Maitra A, Pan D. 2007. Elucidation of a universal size-control mechanism in Drosophila and mammals. Cell 130: 1120-1133.

Dupont S, Morsut L, Aragona M, Enzo E, Giulitti S, Cordenonsi M, Zanconato F, Le Digabel J, Forcato M, Bicciato S, et al. 2011. Role of YAP/ TAZ in mechanotransduction. Nature 474: 179-183.

Eilers M, Schirm S, Bishop JM. 1991. The MYC protein activates transcription of the a-prothymosin gene. EMBO J 10: 133-141.

Fernandez BG, Gaspar P, Bras-Pereira C, Jezowska B, Rebelo SR, Janody F. 2011. Actin-capping protein and the Hippo pathway regulate F-actin and tissue growth in Drosophila. Development 138: 2337-2346.

Guccione E, Martinato F, Finocchiaro G, Luzi L, Tizzoni L, Dall' Olio V, Zardo G, Nervi C, Bernard L, Amati B. 2006. Myc-binding-site recognition in the human genome is determined by chromatin context. Nat Cell Biol 8: 764-770.

Halder G, Dupont S, Piccolo S. 2012. Transduction of mechanical and cytoskeletal cues by YAP and TAZ. Nat Rev Mol Cell Biol 13: 591-600.

Jackson T, Allard MF, Sreenan CM, Doss LK, Bishop SP, Swain JL. 1990. The c-myc proto-oncogene regulates cardiac development in transgenic mice. Mol Cell Biol 10: 3709-3716.

Jansson L, Larsson J. 2012. Normal hematopoietic stem cell function in mice with enforced expression of the Hippo signaling effector YAP1. PLoS One 7: e32013.

Kelly K, Cochran BH, Stiles CD, Leder P. 1983. Cell-specific regulation of the c-myc gene by lymphocyte mitogens and platelet-derived growth factor. Cell 35: 603-610.

Kress TR, Sabo A, Amati B. 2015. MYC: connecting selective transcriptional control to global RNA production. Nat Rev Cancer 15: 593-607.

Kress TR, Pellanda P, Pellegrinet L, Bianchi V, Nicoli P, Doni M, Recordati C, Bianchi S, Rotta L, Capra T, et al. 2016. Identification of MYC-dependent transcriptional programs in oncogene-addicted liver tumors. Cancer Res 76: 3463-3472.

Littlewood TD, Hancock DC, Danielian PS, Parker MG, Evan GI. 1995. A modified oestrogen receptor ligand-binding domain as an improved switch for the regulation of heterologous proteins. Nucleic Acids Res 23: $1686-1690$. 


\section{Croci et al.}

Murphy DI, Junttila MR, Pouyet L, Karnezis A, Shchors K, Bui DA, BrownSwigart L, Johnson L, Evan GI. 2008. Distinct thresholds govern Myc's biological output in vivo. Cancer Cell 14: 447-457.

Pelengaris S, Littlewood T, Khan M, Elia G, Evan G. 1999. Reversible activation of c-Myc in skin: induction of a complex neoplastic phenotype by a single oncogenic lesion. Mol Cell 3: 565-577.

Perna D, Faga G, Verrecchia A, Gorski MM, Barozzi I, Narang V, Khng J, Lim KC, Sung WK, Sanges R, et al. 2012. Genome-wide mapping of Myc binding and gene regulation in serum-stimulated fibroblasts. Oncogene 31: 1695-1709.

Piccolo V, Curina A, Genua M, Ghisletti S, Simonatto M, Sabo A, Amati B, Ostuni R, Natoli G. 2017. Opposing macrophage polarization programs show extensive epigenomic and transcriptional cross-talk. Nat Immunol 18: 530-540.

Roussel MF, Cleveland JL, Shurtleff SA, Sherr CJ. 1991. Myc rescue of a mutant CSF-1 receptor impaired in mitogenic signalling. Nature 353: 361-363.

Sabo A, Kress TR, Pelizzola M, de Pretis S, Gorski MM, Tesi A, Morelli MJ, Bora P, Doni M, Verrecchia A, et al. 2014. Selective transcriptional regulation by Myc in cellular growth control and lymphomagenesis. $\mathrm{Na}$ ture 511: 488-492.

Sansores-Garcia L, Bossuyt W, Wada K, Yonemura S, Tao C, Sasaki H, Halder G. 2011. Modulating F-actin organization induces organ growth by affecting the Hippo pathway. EMBO J 30: 2325-2335.

Shachaf CM, Kopelman AM, Arvanitis C, Karlsson A, Beer S, Mandl S, Bachmann MH, Borowsky AD, Ruebner B, Cardiff RD, et al. 2004. MYC inactivation uncovers pluripotent differentiation and tumour dormancy in hepatocellular cancer. Nature 431: 1112-1117.

Stein C, Bardet AF, Roma G, Bergling S, Clay I, Ruchti A, Agarinis C, Schmelzle T, Bouwmeester T, Schubeler D, et al. 2015. YAP1 exerts its transcriptional control via TEAD-mediated activation of enhancers. PLoS Genet 11: e1005465.

Trumpp A, Refaeli Y, Oskarsson T, Gasser S, Murphy M, Martin GR, Bishop JM. 2001. c-Myc regulates mammalian body size by controlling cell number but not cell size. Nature 414: 768-773.

Vassilev A, Kaneko KJ, Shu H, Zhao Y, DePamphilis ML. 2001. TEAD/TEF transcription factors utilize the activation domain of YAP65, a Src/Yesassociated protein localized in the cytoplasm. Genes Dev 15: 1229-1241.

von Eyss B, Jaenicke LA, Kortlever RM, Royla N, Wiese KE, Letschert S, McDuffus LA, Sauer M, Rosenwald A, Evan GI, et al. 2015. A MYC-driven change in mitochondrial dynamics limits YAP/TAZ function in mammary epithelial cells and breast cancer. Cancer Cell 28: 743-757.

Wada K, Itoga K, Okano T, Yonemura S, Sasaki H. 2011. Hippo pathway regulation by cell morphology and stress fibers. Development 138: 3907-3914.

Walz S, Lorenzin F, Morton J, Wiese KE, von Eyss B, Herold S, Rycak L, Dumay-Odelot H, Karim S, Bartkuhn M, et al. 2014. Activation and repression by oncogenic MYC shape tumour-specific gene expression profiles. Nature 511: 483-487.

Xiao G, Mao S, Baumgarten G, Serrano J, Jordan MC, Roos KP, Fishbein MC, MacLellan WR. 2001. Inducible activation of c-Myc in adult myocardium in vivo provokes cardiac myocyte hypertrophy and reactivation of DNA synthesis. Circ Res 89: 1122-1129.

Yimlamai D, Christodoulou C, Galli GG, Yanger K, Pepe-Mooney B, Gurung B, Shrestha K, Cahan P, Stanger BZ, Camargo FD. 2014. Hippo pathway activity influences liver cell fate. Cell 157: 1324-1338.

Zhao B, Wei X, Li W, Udan RS, Yang Q, Kim J, Xie J, Ikenoue T, Yu J, Li L, et al. 2007. Inactivation of YAP oncoprotein by the Hippo pathway is involved in cell contact inhibition and tissue growth control. Genes Dev 21: 2747-2761 


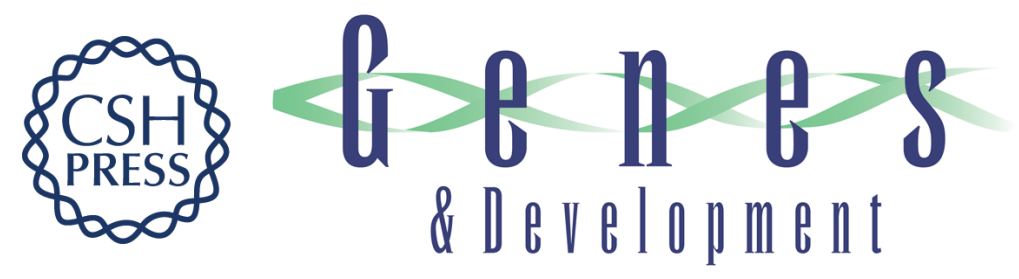

\title{
Transcriptional integration of mitogenic and mechanical signals by Myc and YAP
}

\author{
Ottavio Croci, Serena De Fazio, Francesca Biagioni, et al.
}

Genes Dev. 2017, 31: originally published online November 15, 2017

Access the most recent version at doi:10.1101/gad.301184.117

\section{Supplemental http://genesdev.cshlp.org/content/suppl/2017/11/15/gad.301184.117.DC1 Material}

References This article cites 36 articles, 8 of which can be accessed free at: http://genesdev.cshlp.org/content/31/20/2017.full.html\#ref-list-1
Creative This article, published in Genes \& Development, is available under a Creative Commons Commons License (Attribution 4.0 International), as described at License http://creativecommons.org/licenses/by/4.0/. $\begin{array}{cl}\text { Email Alerting } & \begin{array}{l}\text { Receive free email alerts when new articles cite this article - sign up in the box at the top } \\ \text { right corner of the article or click here. }\end{array}\end{array}$

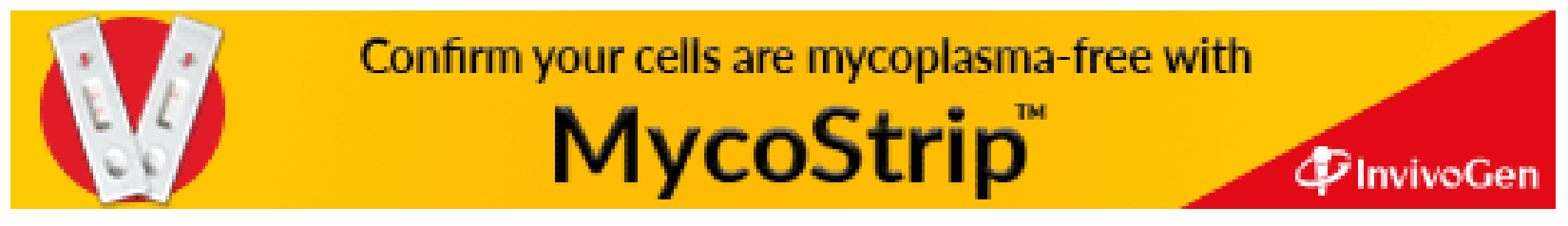

